\title{
Equivocal role of micelles in Eprex adverse events
}

\section{To the editor:}

The news article "Lessons from Eprex for biogeneric firms" in the September issue (Nat. Biotechnol. 21, 956-957) cites an unpublished study conducted by Huub Schellekens and associates that proposes the hypothesis that the stabilizer polysorbate 80 led to the formation of micelles in the formulation of Eprex and that the micelles are a possible cause of pure red cell aplasia associated with Eprex treatment of patients with chronic renal failure.

Unfortunately, the article treats Schellekens' hypothesis as if it were conclusive and exclusive. Schellekens himself does not claim this (see below); he has presented this hypothesis at scientific meetings and readily acknowledges that there are other factors that may also contribute to pure red cell aplasia. Furthermore, he has stated that his hypothesis needs additional work, including testing in animal models. None of this information was included in the news article.

We have consulted with Schellekens and have been supporting his efforts in finding new factors that may underlie the increased incidence of pure red cell aplasia in patients who have been treated with our drug Eprex.

As a matter of public record, it is also well known that cases of pure red cell aplasia have been reported with other recombinant erythropoietins that contain stabilizers other than polysorbate 80 , which would rule out the possibility that micelles are the sole cause, as the article implies.

Since 2001, our company has been conducting a thorough investigation into the causes of erythropoietin-associated pure red cell aplasia and of the factors that have led to more cases in patients treated with Eprex. Our investigation, and the database of knowledge on pure red cell aplasia, clearly point to the causes of the syndrome as multifactorial. Route of administration for patients with chronic renal failure, storage and handling, and changes in the stabilizer used in Eprex have all been identified as potential factors.

We are working closely with health authorities and health-care professionals around the world to better understand and reduce the incidence of pure red cell aplasia. Our efforts to shift the route of administration from subcutaneous to intravenous have borne fruit. In the first seven months of 2003, we have seen a marked decline in reported cases of the condition. Worldwide, the incidence has dropped from 3.16 cases per 10,000 patient years in the first half of 2002 to 0.43 cases per 10,000 patient years in the first half of 2003.

In addition, we are working on several hypotheses regarding the formulation of Eprex, including the micelle hypothesis. We welcome all responsible scientific inquiry into this issue. However, we also expect a scientifically accurate rendering of all the facts when news of a study appears in a wellrespected journal such as Nature Biotechnology.

\section{Janice M Smiell}

Johnson \& Johnson Pharmaceutical Research \& Development, Senior Director/Clinical Leader, Global Development, 920 Route 202, P.O. Box 300, Raritan, NJ 08869, USA. e-mail:jsmiell@prdus.jnj.com

\section{To the editor:}

As the senior authors referenced in the news article "Lessons from Eprex for biogeneric firms" (Nat. Biotechnol. 21, 956-957), we would like to point out that the news article draws conclusions that are not supported in the paper entitled "Micelle-associated protein in epoetin formulations" that is in press at the journal Pharmaceutical Research.

The opening line of the news article states: "Dutch scientists have found that aggregates of small molecules (micelles) in the formulation of erythropoietin alpha (EPO), sold as Eprex in Europe, were responsible for an immunogenic reaction that triggered severe side effects". In the third paragraph, the article further states: "But the high concentration of the sorbitol [polysorbate 80] led to the formation of micelles, which were found to cluster with EPO in a form that triggers an immunogenic reaction. The resulting antibodies not only abrogated the effects of Eprex, but also neutralized naturally occurring EPO."
The above statements mistakenly imply that the unpublished research paper has provided definitive proof for the cause of erythropoietinassociated pure red cell aplasia in patients with chronic renal failure. In the Pharmaceutical Research paper, we and our coauthors clearly present the development of an important hypothesis that requires further study. For example, we state that "this micellar form of epoetin may be an important risk factor for the development of antibodies in patients," adding "we recognize that the presence of micelle-associated epoetin as a risk factor for immunogenicity in patients at the moment remains hypothetical" and "Follow-up studies in which the micelle-associated epoetin is tested in animal models may shed light on the correlation between the formulation of epoetin and its immunogenicity."

We make no claim to a direct cause-effect relationship between the micelle-associated epoetin and immunogenicity. We only propose that such an interaction could be possible and deserves serious further investigation. Although the work published in the Pharmaceutical Research article was not supported by the manufacturer of Eprex, we are now collaborating with the company (Johnson \& Johnson, Raritan, NJ, USA) to further investigate our initial results. We have joint analytical efforts ongoing to confirm and better characterize the nature of the micelleassociated erythropoietin, and we will be conducting animal studies to explore the immunogenicity of the complex. This collaboration will allow us to obtain verification of results in separate and independent laboratories.

\section{Huub Schellekens}

Director, Central Animal Institute, Utrecht University, P.O. Box 80190, 3508 TD Utrecht, The Netherlands

\section{Daan J A Crommelin}

Department of Pharmaceutics, Utrecht Institute for Pharmaceutical Sciences, Utrecht University, P.O. Box 80082, 3508 TB Utrecht, The Netherlands

e-mail:d.j.a.crommelin@pharm.uu.nl 